\title{
Edge Modification of Graphene Using Concurrent High Temperature Heating and Electron Beam Irradiation
}

\author{
Herman C. Floresca*, Stephen Mick**, Ning Lu*, Jinguo Wang* and M. J. Kim* \\ * Department of Materials Science and Engineering, University of Texas, Dallas, Richardson, TX \\ 75080 \\ **Protochips, Inc. Raleigh, NC 27606
}

Graphene, which has a single atomic layer of carbon atoms bonded in a hexagonal lattice, is among the few materials that are stable in two dimensions and free-standing when suspended. With one dimension confined, the other two dimensions may hold the key in determining its unusual properties. Studies show that the edge structure of a graphene ribbon or flake has a major impact on their electronic properties. Control of this edge structure is difficult during synthesis or exfoliation so a post treatment may be the most viable option. It has been reported that pinholes in graphene layers became larger with time under irradiation of an $80 \mathrm{kV}$ electron beam [1]. The energy needed to remove a carbon atom at the edge of a lattice is less than what is needed to remove one that is fully saturated with surrounding atoms, thus the sputtering is more likely to happen along the edges than the middle of a flake. Huang and co-workers reported that heat treatment along with a high electron beam irradiation energy of a flake sublimates and reconstructs its edges when there are multiple layers present [2]. Using a $300 \mathrm{kV}$ TEM and Joule heating to $\sim 2000^{\circ} \mathrm{C}$, two graphene monolayer edges can reconstruct into one bi-layer edge, similar to a curled or folded single edge. This configuration was believed to be energetically favorable compared to two monolayers with dangling carbon atoms and appeared to be more stable and resistant to sputtering under the high energy of the electron beam.

In this study, we try to use both sputtering and reconstruction to controllably modify the edge of a graphene flake. The samples were prepared by manually grinding small highly ordered pyrolytic graphite (HOPG) blocks into fine powders and dispersing in alcohol. The solution was then drop cast onto a conductive ceramic membrane (or thermal E-chip) provided by Protochips. The holder allows for specimen heat treatments up to $1200^{\circ} \mathrm{C}$ with millisecond heating rates in-situ of our JEOL JEM$2100 \mathrm{~F}$ field emission electron microscope.

Fig. 1(a) shows a high resolution TEM (HRTEM) image of the edge of a graphene flake which contains multiple mono-layers with very rough edges and contaminants. The flake is heated to $400^{\circ} \mathrm{C}$. Images of the flake were taken at this starting temperature since without the heater, the high voltage of the electron beam would cause the graphene layers' lattice to turn disordered and amorphous if imaged too long [2]. When heated to $800^{\circ} \mathrm{C}$, in Fig. 1(b), the contaminants begin to disappear off the surface of the graphene. The edges also begin to sputter away and reconstruct, distinguished by the higher contrast lines in the image. Once two monolayer edges reconstruct together to form a bi-layer edge, it becomes more resistant to the sputtering and stabilizes. The bilayer edges also form at $60^{\circ}$ preferences to each other, due to the hexagonal structure of the graphene lattice. At $1200^{\circ} \mathrm{C}$, the contamination has disappeared leaving a clear set of graphene layers in Fig. 1(c). Large holes form on some of the single layers in the flake as a result of the high energy of the electron beam. These holes stop growing once the edge of the hole reconstructs into a bi-layer. At 
this temperature, the edge of the flake begins to reconstruct into almost atomically straight structures as a result of the concurrent electron beam sputtering and high temperature reconstruction.

In Summary, the edge of the multi-layer graphene flake can be modified by two mechanisms that are occurring at the same time: the sputtering away of the lattice by the electron beam and the reconstruction of two monolayer edges into a bi-layer edge through high temperatures. This modifying process allows us to straighten out a multilayer flake edge on an atomic scale.

\section{References}

[1] J. Warner et al., Nature Nanotech., 4 (2009) 500.

[2] J. Huang et al., Proc. Natl. Acad. Sci., 106 (2009) 10103.

[4] This work was supported by a grant from the State of Texas ETF (FUSION), SWAN (GRCNRI) and AOARD-AFOSR (FA2386-10-1-4066).

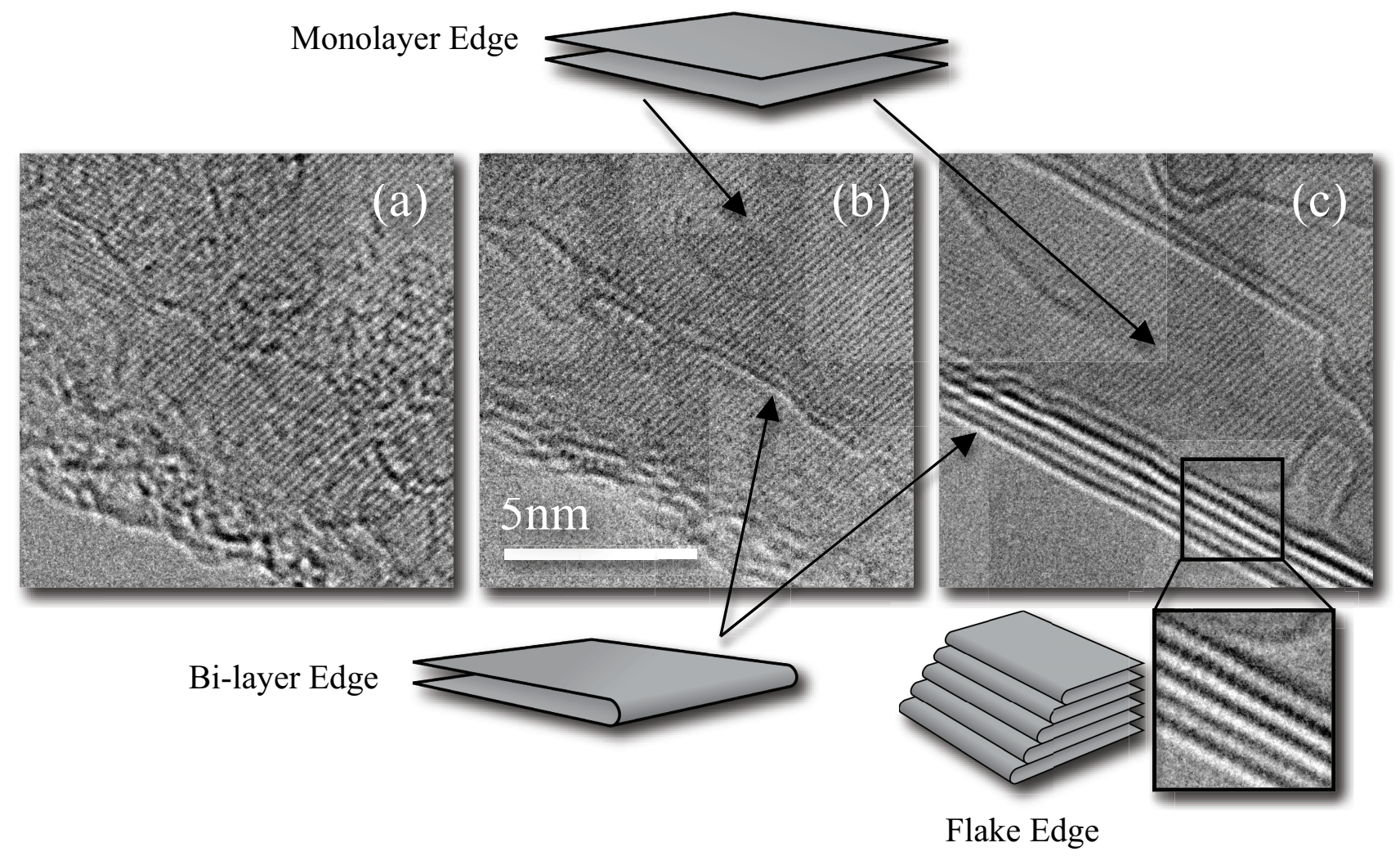

FIG. 1. HRTEM images of the graphene flake's edge under different temperatures. Schematic illustrations are given for monolayer edge structures, bi-layer edge structures and the flake edge. (a) At $400^{\circ} \mathrm{C}$ the flake is contaminated and has a very rough and jagged edge. (b) At $800^{\circ} \mathrm{C}$ the contaminants are disappearing and edges of the graphene layers are being etched. (c) At $1200^{\circ} \mathrm{C}$ the edges are reconstructing into bi-layer edges, distinguished by higher contrasts compared to monolayer edges. The combination of etching and reconstruction modifies the edge of the graphene flake into atomically straight structures. 